\title{
Measurement of Phased Array Point Spread Functions for use with Beamforming
}

\author{
Chris Bahr, Nikolas S. Zawodny, ${ }^{\dagger}$ Brandon Bertolucci, ${ }^{\dagger}$ Kyle Woolwine, ${ }^{\ddagger}$ \\ Fei Liu, ${ }^{\S}$ Jian Li, ${ }^{* *}$ Mark Sheplak, ${ }^{\dagger \dagger}$ and Louis Cattafesta ${ }^{\dagger \dagger}$ \\ Florida Center for Advanced Aero-Propulsion \\ Interdisciplinary Microsystems Group \\ University of Florida, Gainesville, FL 32611
}

\begin{abstract}
Microphone arrays can be used to localize and estimate the strengths of acoustic sources present in a region of interest. However, the array measurement of a region, or beam map, is not an accurate representation of the acoustic field in that region. The true acoustic field is convolved with the array's sampling response, or point spread function (PSF). Many techniques exist to remove the PSF's effect on the beam map via deconvolution. Currently these methods use a theoretical estimate of the array point spread function and perhaps account for installation offsets via determination of the microphone locations. This methodology fails to account for any reflections or scattering in the measurement setup and still requires both microphone magnitude and phase calibration, as well as a separate shear layer correction in an open-jet facility. The research presented seeks to investigate direct measurement of the array's PSF using a non-intrusive acoustic point source generated by a pulsed laser system. Experimental PSFs of the array are computed for different conditions to evaluate features such as shift-invariance, shear layers and model presence. Results show that experimental measurements trend with theory with regard to source offset. The source shows expected behavior due to shear layer refraction when observed in a flow, and application of a measured PSF to NACA 0012 aeroacoustic trailing-edge noise data shows a promising alternative to a classic shear layer correction method.
\end{abstract}

\section{Introduction}

TN aeroacoustic wind tunnel testing, phased microphone arrays have become common instrumentation. Phased

array techniques apply a source model to data measured from a sensor array and generate a map of estimates of source locations and strengths [1]. Unfortunately, the conventional beamformer output using the delay-and-sum technique, or any variant thereof, is convolved with the array's point spread function [2]. This point spread function (PSF) arises due to the array's finite aperture and finite sample locations, and is a representation of the array's output response to an ideal point source at a given location in space. The PSF is a function of frequency and, except for the special case of a plane wave field, is not shift-invariant to the source location.

Research Aerospace Engineer, Aeroacoustics Branch, NASA Langley Research Center, Hampton, VA 23681, Member AIAA (Formerly Post-Doctoral Research Associate at University of Florida, where research was conducted)

$\dagger \quad$ Graduate Research Assistant, MAE Department, P.O. Box 116250, Student Member AIAA

* Undergraduate Research Assistant, MAE Department, P.O. Box 116250, Student Member AIAA

$\S \quad$ Research Scientist, MAE Department, P.O. Box 116250, Member AIAA

** Professor, ECE Department, P.O. Box 116200

$\dagger$ Professor, MAE Department, P.O. Box 116250, Associate Fellow AIAA, cattafes@ufl.edu

1

American Institute of Aeronautics and Astronautics 
Multiple methods exist to attempt to remove the effects of the PSF from a beamformer output, or beam map. A non-exhaustive list of the current methods in use includes DAMAS [3], DAMAS2 [4], FFT-NNLS [5], CLEAN-SC [6], CMF [7], LORE [8] and MACS [9]. DAMAS, CMF, LORE and MACS formulate the deconvolution problem as the solution to a large system of equations. These methods allow for a variable PSF through varying steering vector terms but may be computationally intensive depending on the problem of interest. DAMAS2 and FFT-NNLS treat the deconvolution problem as an image processing technique and attempt to iteratively divide out the effects of the PSF in a Fourier transform of the original beam map and PSF, similar to the Weiner Filter [4]. The original formulation of these requires a shift-invariant PSF, but the algorithms can be modified to allow variation $[5,10]$. Shift-invariance assumes that the PSF's shape does not change with source location, and that the overall beam pattern is simply shifted in space [4]. CLEAN-SC is the only method from those listed above which does not use an explicit deconvolution approach, instead modifying the beam map to remove coherent sidelobe contributions.

For all of the mentioned methods that use the PSF, an ideal theoretical PSF based on the array design is generally used. Array calibration, both individual and group, is handled before deconvolution occurs. Individual channel calibration, compensating for magnitude and phase offsets in each element of the array, is often done ahead of time and applied in the construction of the cross-spectral matrix, prior to beamforming. Group calibration can alternatively correct for microphone magnitude and phase offsets and compensate for small positional errors in microphone placement when shift-invariance is assumed. This technique can correct the steering vectors used in conventional beamforming [2]. Corrections for shear layers, when conducting experiments in open-jet wind tunnels, [11] are also applied using the conventional steering vectors. None of these methods directly account for real installation effects such as reflections and scattering, which alter the appropriate Helmholtz equation solution (as all of these analysis techniques apply to the frequency domain free-field boundary conditions).

The PSF is generally treated as the array's response function to a point source input, often observed in the frequency domain [4]. It can also be considered as the array's observation of the response function of a test setup [13] for a particular source location, similar to a single observation of the installation's Green's function. In this sense, the theoretical PSF with standard corrections applied may fall short of truly characterizing the observed array response for a point source. Due to the computational complexity of determining the point source response of a real experimental setup for all frequencies of interest, experiments provide the most promising route for evaluating the true PSF.

Some previous research has been conducted regarding experimental analysis of PSFs. PSF analysis and deconvolution have a long history in the fields of optics and radio astronomy [4]. Dougherty's work on group calibration can be considered an approximation of a measurement of a free-space Green's function [2]. Dougherty also discussed the effects of reverberant walls in a reverberant facility on phased array output. Oerlemans and Sijtsma experimentally studied the effect of reverberant sidewalls in an open jet wind tunnel on phased array output [12]. Fenech and Takeda measured the appropriate Green's function for a reverberant facility to compare with an image source correction to the free-space Green's function [13]. In both cases, computing an in-situ experimental correction improved beamforming performance. Both of these experiments, however, used intrusive acoustic sources which precluded measurements from occurring when test section flow was present. The present study, in contrast, uses a non-intrusive analysis method which can be used both with and without flow, allowing shear layer corrections to be experimentally incorporated into approximate point spread functions [14].

This paper first presents the development of the theoretical PSF for a spherical wave beamformer. It then describes how an experimental PSF measurement can be used with image-processing based deconvolution and in the formulation of experimental steering vectors. The experimental setup is then described for measuring an acoustic point source generated by a pulsed laser system under different conditions. Results are presented for varying 
experimental conditions, including different physical facility and model configurations, as well as mean flow effects, before being used to correct an aeroacoustic measurement. Conclusions and future work follow.

\section{Theoretical Development and Implementation}

In phased array analysis, a microphone array, like the one shown in Figure 1 and Figure 2, is used to sample an acoustic field in space and time. The data from the array measurement are acquired and, for conventional beamforming and all its derivatives, averaged to obtain auto- and cross-power estimates in the form of a crossspectral matrix (CSM). Consider an $M$-element array, with subscript $m$ denoting a given microphone within the array, and subscript 0 denotes the array center at Cartesian origin $(0,0,0)$. For a given frequency, the conventional beamforming equation for power $P$ from scan point $l$ using a CSM is given by [15]

$$
P_{l}=\frac{1}{M^{2}} \vec{a}_{l}^{H} \vec{G} \vec{a}_{l}, \quad l=1, \ldots L .
$$

Here,

$$
\vec{a}_{l}=\frac{1}{r_{l, 0}}\left[r_{l, 1} e^{j k r_{l, 1}} \cdots r_{l, M} e^{j k r_{l, M}}\right]^{T}
$$

is the steering vector corresponding to the $l^{\text {th }}$ scanning point within the region of interest. The term $r_{l, m}$ is the distance from the $l^{\text {th }}$ scanning point to the $m^{\text {th }}$ microphone (or array center in the case of $m=0$ ), $k$ is the acoustic wavenumber, and the $M \times M$ matrix $\vec{G}$ is the CSM. Note that $(.)^{T}$ and $(.)^{H}$ denote the transpose and conjugate transpose operations, respectively. This steering vector is selected such that the beamformer output, when steered to a correct source location in a single-source spherical-wave acoustic field, corresponds to the acoustic power measured at the center of the array due to the source [3]. The PSF of an array can be considered the normalized output of Equation (1) when a point source is present at scan point $l_{i}$, and no other sources are present. This can be expressed where, in a 2-D scan plane a distance $z_{s}$ from the array face, $l_{i}$ is located at $\left(0,0, z_{s}\right)$.

For image-processing based deconvolution methods, an image map of the PSF along with the experimental delay-and-sum beam maps are the necessary inputs. The PSF map is often theoretically constructed based on the theoretical free-field response of an array, but nothing prevents it from being an experimental measurement. Example theoretical PSFs for the array shown in Figure 1 and Figure 2 are shown in Figure 3 and Figure 4. Note that the spiral in Figure 2 is discontinuous on the inner ring due to a design modification, and the array is designed for minimal sidelobes $10 \mathrm{~dB}$ down, but plotted on a $15 \mathrm{~dB}$ scale to accentuate response behavior.

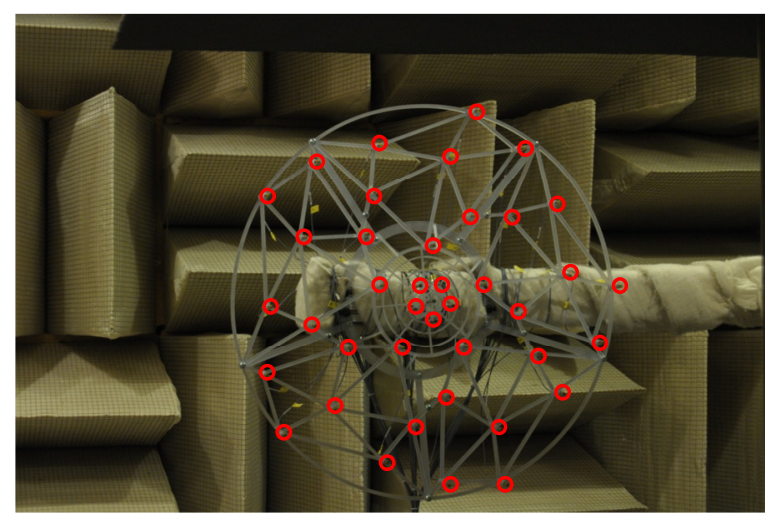

Figure 1. Example free-field microphone array, prior to application of acoustic treatment.

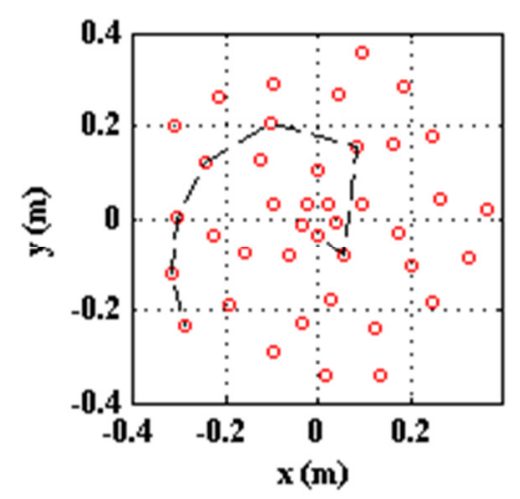

Figure 2. Microphone coordinates for array from Figure 1. 


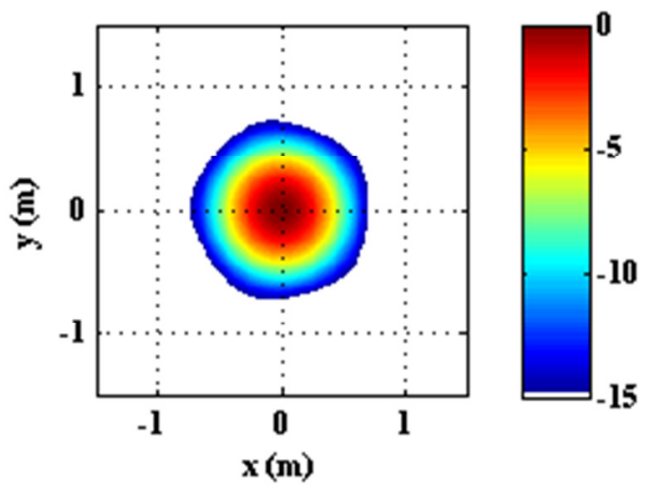

Figure 3. Theoretical $1 \mathrm{kHz}$ PSF (source-to-array distance of $1.13 \mathrm{~m}$ ) for array from Figure 1 in $\mathrm{dB}$ ref. peak.

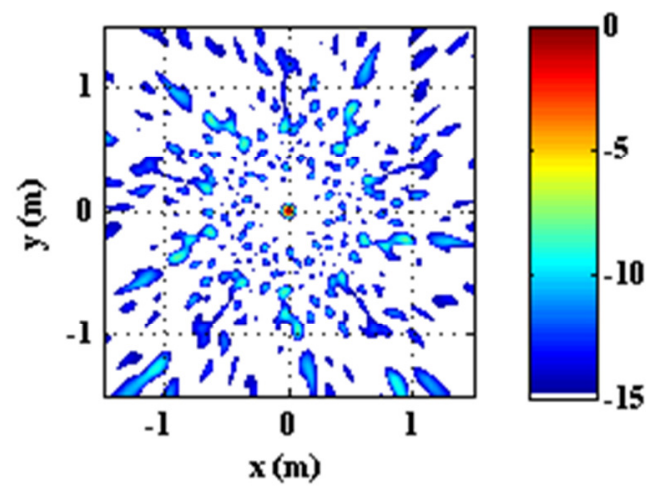

Figure 4. Theoretical $10 \mathrm{kHz}$ PSF (source-to-array distance of $1.13 \mathrm{~m}$ ) for array from Figure 1 in $\mathrm{dB}$ ref. peak.

For image-processing based deconvolution, a map of an array PSF using the free-space steering vectors from Equation (2), either generated from theory or using an experimental data set, can provide sufficient information for deconvolution. However, methods such as DAMAS traditionally use modeled steering vectors rather than directly modeled PSFs. If experimental PSF measurements are to be incorporated into these methods, the experimental data must be used to construct experimental steering vectors, modify free-space steering vectors, or correct the data CSM [16]. Below, an idealized point source measurement is discussed. This ideal measurement allows for formulation of potential steering vector correction methods, similar to the calibration methods of Dougherty [2] and Brooks et al. [16].

The power-based methodology from Equation (1) is necessary because aeroacoustic measurements are observations of stochastic fields. However, for discussion of steering vector corrections, formulation of the problem as a noiseless measurement of a deterministic signal is instructive. Under these simplifying conditions the complex pressure output of a simple, frequency-domain beamformer can be expressed using matrix multiplication as $[1,14]$

$$
p_{l}=\frac{1}{M} \vec{y} \vec{a}_{l} .
$$

Here the row vector $\vec{y}$ contains the complex data for a given frequency bin of the Fourier transforms of the measured microphone signals,

$$
\vec{y}=\left[\begin{array}{lll}
y_{1} & \cdots & y_{M}
\end{array}\right] .
$$

From this statement, the individual contribution of each microphone to the beamformer output is thus quantified by the multiplication of the microphone output by a steering coefficient

$$
p_{l, m}=y_{m} \frac{r_{l, m}}{r_{l, 0}} e^{j k k_{l, m}} .
$$

The farfield radiation from a monopole source at location $l$ can be expressed as [17]

$$
y(r)=A_{l} \frac{1}{r} e^{-j k r},
$$

where $r$ is the distance from the point source location to the observer location and $A_{l}$ is a model source strength.

Substituting Equation (6) into Equation (5) for the appropriate microphone locations gives

$$
p_{l, m}=A_{l} \frac{1}{r_{l, m}} \frac{r_{l, m}}{r_{l, 0}} e^{j k_{l, m}-j k l_{l, m}}=\frac{A_{l}}{r_{l, 0}} .
$$

Under ideal free-space conditions, the steering coefficient correctly collapses the data to the model source strength, scaled to the array center location. Of course, this is rarely the case. In reality, a time-harmonic acoustic field is expressed as 


$$
y(\vec{r})=B_{l}(\vec{r}) e^{j \theta(\vec{r})},
$$

where source spherical symmetry is no longer enforced, and acoustic scattering and reflection can occur. Here, $\theta(\vec{r})$ represents the general phase angle of the acoustic field at every point in space. Similarly,

$$
y_{m}=B_{l, m} e^{j \theta_{m}}
$$

is a general expression of the output of the $m^{\text {th }}$ microphone, which can now include deviations in the microphone position from its ideal location, as well as magnitude and phase shifts in the signal due to the microphone's real frequency response. Applying the ideal free-space steering coefficient to Equation (9) provides no guarantee of properly scaling the data.

For a known source, the steering vector could be correctly computed in an experiment by measuring the magnitude and phase shift from the source to each microphone. Unfortunately, the precise magnitude and phase of most compact acoustic sources is immeasurable without an intrusive experimental setup. However, an alternate approach can be employed by placing a microphone at the array center as a reference measurement. Conceptually, the steering coefficient determines the phase angle of the acoustic field measured at a given microphone relative to the phase angle of the field at the source location. Equation (5) can thus be re-stated as

$$
p_{l, m}=y_{m} \frac{r_{l, m}}{r_{l, 0}} e^{j\left(\theta_{l}-\theta_{m}\right)} .
$$

If the phase angle is subsequently shifted from the source to the array center, Equation (10) becomes

$$
p_{l, m}=y_{m} \frac{r_{l, m}}{r_{l, 0}} e^{j\left(\theta_{l}-\theta_{m}\right)} e^{j\left(\theta_{0}-\theta_{l}\right)}=y_{m} \frac{r_{l, m}}{r_{l, 0}} e^{j\left(\theta_{0}-\theta_{m}\right)} .
$$

In the case of a free-space measurement where the acoustic field takes the form of Equation (6), the measurement at an array microphone is

$$
y_{m}=\frac{A_{l}}{r_{l, m}} e^{-j k r_{l, m}}
$$

while the measurement at the reference microphone is

$$
y_{0}=\frac{A_{l}}{r_{l, 0}} e^{-j k r_{l, 0}} \text {. }
$$

Dividing Equation (13) by Equation (12) yields the frequency response between the reference microphone and the array microphone,

$$
H_{0, m}^{l}=\frac{y_{0}}{y_{m}}=\frac{A_{l}}{A_{l}} \frac{r_{l, m}}{r_{l, 0}} e^{-j k\left(r_{l, 0}-r_{l, m}\right)}=\frac{r_{l, m}}{r_{l, 0}} e^{j\left(\theta_{0}-\theta_{m}\right)} .
$$

Thus, the steering term of Equation (11) is simply the in-situ frequency response between the two microphones, and

$$
p_{l, m}=H_{0, m}^{l} y_{m}
$$

Note that in this model a constant phase angle offset, $\theta_{0}$, is present in the frequency response, Equation (14). When such steering vectors are constructed for Equation (1), this offset cancels through the multiplication of the conjugate transpose. This frequency response function, $H_{0, m}^{l}$, is a function of microphone location and performance, array center location, source location, and installation effects for a given experimental setup. This methodology is straightforward to implement experimentally by including a reference microphone at the array center and is consistent with existing calibration techniques [2]. Assuming the reference microphone is calibrated to a known standard, this steering coefficient should correct for non-idealities in the array microphone's response, as well as positional uncertainties, shifts due to shear layer interactions, scattering and reflections. This can be illustrated by again using the free-space monopole model from Equation (12). Here, 


$$
H_{0, m}^{l}=\frac{r_{l, m}}{r_{l, 0}} \frac{e^{-j k r_{l, 0}}}{e^{-j k r_{l, m}}}
$$

and

$$
p_{l, m}=y_{m} H_{0, m}^{l}=\left(\frac{A_{l}}{r_{l, m}} e^{-j k r_{l, m}}\right)\left(\frac{r_{l, m}}{r_{l, 0}} \frac{e^{-j k k_{l, 0}}}{e^{-j k r_{l, m}}}\right)=\frac{A_{l}}{r_{l, 0}} e^{-j k r_{l, 0}},
$$

recovering the expected behavior from Equation (7) with the additional expected phase offset.

Unfortunately, as mentioned above, this response function is dependent on source location, so strictly speaking an experiment would need to be conducted with a point source sequentially traversed through every scan point in $L$, and the corrected steering vector would be

$$
a_{l, c o r r}=\left[\begin{array}{lll}
H_{0,1}^{l} & \cdots & H_{0, M}^{l}
\end{array}\right]^{T} .
$$

If such a measurement were conducted then this library of steering vectors could be used directly for beamforming and potentially deconvolution. However, since large scan grids of interest make this type of measurement intractable, this response function must instead be developed as an approximate correction to the standard free-space steering vector. A similar correction method currently exists in the literature [2]. If shift-invariance is assumed, which is the case when array-to-scan-plane distances are much greater than the array aperture and the scan plane dimensions [4], the correction factor is independent of the source location (also assuming ideal, omnidirectional, non-intrusive microphone measurements). For instance, if the response function for a centered source is defined as $H_{0, m}^{L / 2}$, i.e. the geometric center of the scan plane corresponds to the middle scan point index, then the corrected steering vector could be constructed as

$$
a_{l, c o r r}=\frac{r_{L / 2,0}}{r_{l, 0}}\left[r_{l, 1} \frac{H_{0,1}^{L / 2}}{r_{L / 2,1} e^{j k k_{L / 2,1}}} e^{j k k_{l, 1}} \cdots r_{l, M} \frac{H_{0, M}^{L / 2}}{r_{L / 2, M} e^{j k r_{L / 2, M}}} e^{j k r_{l, M}}\right]^{T} .
$$

When $l=L / 2$, Equation (19) reduces to Equation (18). If this method is applied to a data set with no flow in an anechoic enclosure, the correction factor conceptually reduces to the array calibration technique discussed by Dougherty [2]. The method can also be used to calibrate through a shear layer on a frequency-by-frequency basis, effectively the same way as presented by Brooks et al. [16]. As mentioned by those authors, additional corrections are required for temperature differences between calibration measurements and aeroacoustic experiments. This correction method assumes a non-intrusive, ideal measurement from the center reference microphone. So, for instance, directive scattering of a center free-field microphone at high frequencies would require additional field modeling and correction. Scattering interactions between the center microphone and array microphones would compound the problem.

To account for varying steering vectors in space, the source can be placed at several locations within the test section. The results of these individual measurements could, for this most simple methodology, be used to interpolate and extrapolate a response function surface across the scan plane. Such analysis is beyond the scope of this paper, although some research has been conducted by Suzuki which may be applied in this venue [10]. In summary, by maintaining a reference microphone at an observation point of interest (the array center), a point source can be used to determine what the relationship is between an array microphone and the reference microphone, and compare that relationship to the modeled free-space relationship. This can be used ideally in place of the modeled steering vectors, or as a lower-order model [16] as a correction to the existing steering vectors or data. 


\section{Experimental Setup}

A non-intrusive, laser-based acoustic point source is used to directly measure the point spread function of an array in the University of Florida Aeroacoustic Flow Facility (UFAFF). This type of source has been used previously in UFAFF [14]. Since this previous work, improvements have been made to the source.

\section{A. Acoustic Source}

An acoustic point source is generated using a pulsed laser system. The selected laser is a New Wave Solo 120XT dual Nd:YAG system, capable of outputting a maximum instantaneous energy of $120 \mathrm{~mJ} / \mathrm{pulse}$ at a wavelength of $532 \mathrm{~nm}$, with a nominal pulse width of 3-5 ns. The laser beam is expanded and re-focused through a multi-stage lens telescope assembly, the components of which are detailed in Figure 5. As shown, a total of four lenses are used. The first two are singlet plano-concave lenses, and expand the incident laser beam. The second two are achromatic doublet lenses, and re-focus the laser beam. All lenses are made from BK 7 glass and have a 532-nm narrowband anti-reflective coating, minimizing energy losses through the telescope assembly. The telescope housing consists of a series of $50.8 \mathrm{~mm}$ long black-anodized aluminum tubes.

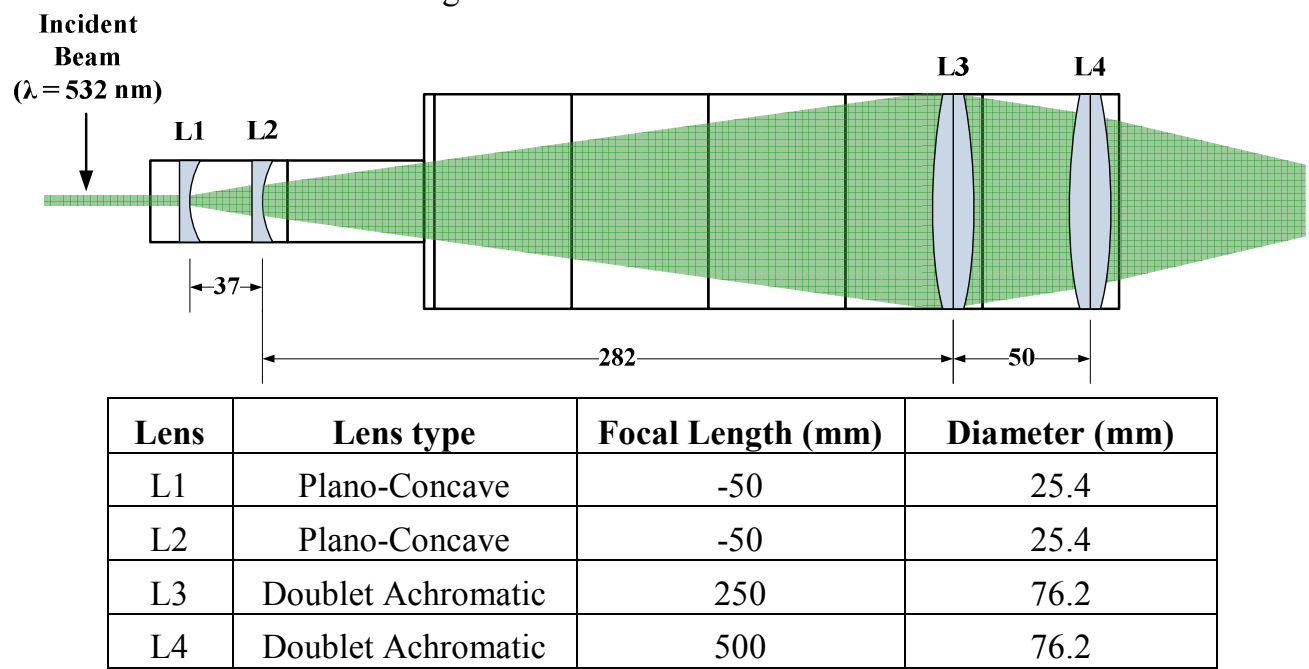

Figure 5. Schematic of lens telescope assembly. Dimensions in schematic are in $\mathbf{m m}$.

The lens telescope assembly increases the solid angle of convergence of the laser beam, in an effort to minimize the resulting focal spot area, which was found to be one of the deficiencies of the previous point source installation in UFAFF. The arrangement is chosen first to achieve a mean pulse intensity which allows for reliable pulse source formation and second to position the source location near the center of the UFAFF test section. Previous literature suggests that a minimum pulse intensity on the order of $10^{11} \mathrm{~W} / \mathrm{cm}^{2}$ is necessary for an acoustic source to form in air [18]. A Gaussian quality factor of approximately 30 is estimated based on observation of the divergence of the incident laser beam. This yields an effective focused laser spot size of $111 \mu \mathrm{m}$ and a resulting peak intensity of approximately $2 \times 10^{11} \mathrm{~W} / \mathrm{cm}^{2}$ [19]. This is found to produce an acoustic source in free air, but not reliably. In order to increase the success rate of source formation, a Tektronix dual-channel function generator is used to overlap, with a precision on the order of nanoseconds, the firing of the second laser pulse of the 120XT system with the first through the laser triggering controls. This dramatically increases the source formation success rate in quiescent air, with an optimum repetition rate of $1 \mathrm{~Hz}$. However, installation vibration at higher test section Mach numbers reduces the success rate dramatically, so flow seeding is introduced for with-flow measurements. Unlike with the previous work [14], the seeding does not appear to affect the consistency of source formation timing, possibly due to the reduced spot size of $111 \mu \mathrm{m}$ of the laser focal point. 


\section{B. Acoustic Instrumentation}

A new acoustic array has been designed and fabricated for use with UFAFF [20]. This array frame, shown in Figure 1, consists of a small-aperture $(7.62 \mathrm{~cm})$ array nested in a large-aperture $(73.7 \mathrm{~cm})$ array, with a total of 61 microphones present on the array frame. Each array was initially designed as a zero-redundancy multi-arm logarithmic spiral array [21]. The center reference microphone of the array is a B\&K $49541 / 4$ " free-field microphone, which has a nominal $\pm 3 \mathrm{~dB}$ frequency range of $4 \mathrm{~Hz}-100 \mathrm{kHz}$. The inner two rings of the array are also each populated with five B\&K 4954 microphones. The next three rings of the array are each populated with five G.R.A.S. 40BE 1/4" microphones, which also have a $\pm 3 \mathrm{~dB}$ frequency range of $4 \mathrm{~Hz}-100 \mathrm{kHz}$. These first five rings define the inner small-aperture array. The remaining seven rings each consist of five B\&K $49581 / 4$ " microphones with a $\pm 3 \mathrm{~dB}$ frequency range of $10 \mathrm{~Hz}-20 \mathrm{kHz}$. These outermost seven rings, along with the outermost of the three G.R.A.S. rings, define the outer large-aperture array. For all array experiments, all of the microphones are installed with their protective grids.

While the sampling rate and microphone bandwidth allow for higher-frequency analysis, data are only considered up to $10 \mathrm{kHz}$ due to scattering effects. As mentioned in Section II, the methods discussed assume a nonintrusive, or at least correctible, reference microphone measurement. The reference microphone in this case is within approximately one microphone diameter to the inner array microphones on the array frame, and a significant scattering effect is noticeable at higher frequencies as shown in subsequent sections. This is verified by subsequent single free-space microphone measurements. Acoustically absorbent foam is applied to the face of the array to mitigate this scattering effect, but to simplify analysis and discussion, results are only considered in a band where scattering is not a problem and acoustic treatment is unnecessary. The array pattern of the outer array is shown in Figure 2, with example theoretical PSFs at $1 \mathrm{kHz}$ and $10 \mathrm{kHz}$ shown in Figure 3 and Figure 4, respectively.

As a subset of this research, individual free-space measurements are also conducted to evaluate source behavior, as well as inter-microphone scattering from the inner array microphones to the reference microphone. For these experiments the array is removed from the facility, and a single microphone located in free space where the array center reference microphone is normally located, relative to the source. A B\&K 4939 1/4" free-field microphone, with a $\pm 2 \mathrm{~dB}$ frequency range of $4 \mathrm{~Hz}-100 \mathrm{kHz}$, is first installed with its protective grid. The grid is then removed to evaluate grid effects on the observed signal. A B\&K 4138 1/8" pressure-field microphone with a $\pm 2 \mathrm{~dB}$ frequency range of $6.5 \mathrm{~Hz}-140 \mathrm{kHz}$ is then installed, with grid, in place of the B\&K 4939 microphone to evaluate additional power in the signal beyond $100 \mathrm{kHz}$. Setup permitting, certain configurations have one or two B\&K 4939 reference microphones located equidistant from the acoustic point source, on the opposite side of the test section.

\section{Data Acquisition}

Data are acquired using an 18-slot National Instruments PXI-1045 chassis. For most experiments, the chassis is populated with 17 NI PXI-4462 data acquisition (DAQ) cards. Each channel has 24-bit resolution with $118 \mathrm{~dB}$ dynamic range. The first 60 channels are connected to the array microphones. The $61^{\text {st }}$ channel is connected to the center reference microphone. Depending on configuration, the next one or two channels are occupied by additional reference microphones. For laser source cases, a Thorlabs DET10A photodetector is connected as the last acquistion channel to determine the source initiation time and thus acoustic transit times. All acoustic measurements are ac coupled with a $-3 \mathrm{~dB}$ cut-on at $3.4 \mathrm{~Hz}$, and appropriate anti-aliasing filters are automatically applied. The photodetector channel is dc coupled. The sampling rate when using these cards is set to 204,800 samples per second. All channels are acquired simultaneously.

When acquiring laser pulse data, acquisition is triggered from the output of the laser Q-switch. For each channel, 6400 samples are acquired, for a resultant block length of $1 / 32^{\text {nd }}$ of a second, and 1000 such blocks of data 
are acquired. Because the Q-switch fires whether or not an acoustic source forms, a block rejection scheme is necessary in post-processing as described in [14]. Aeroacoustic data are acquired with the same sampling parameters, but continuously for 30 seconds.

As previously mentioned, source behavior is evaluated using microphones installed in free space for comparison with the array center reference microphone. As a subset of this work, a high-speed DAQ card is used to investigate the effects of finite sampling rates on the observed signal. A single, 2-channel 14-bit NI PXI-5122 DAQ card is installed in the chassis, and connected to either the array center reference microphone or a free-space microphone, depending on experimental configuration. The photodetector is connected to the second channel. Data are simultaneously acquired at a sampling rate of 100 megasamples per second. Acoustic channels are ac coupled with a $-3 \mathrm{~dB}$ cut-on at $12 \mathrm{~Hz}$. Acquisition is again triggered from the laser Q-switch. System throughput limits the block length to $1 / 64^{\text {th }}$ of a second. As with other laser pulse cases, 1000 blocks are acquired, and a rejection scheme is necessary to detect blocks where no acoustic source is present.

\section{Data Processing}

As described in [14], laser pulse data blocks are ensemble averaged in the time domain to reduce the influence of random electronic and aerodynamic noise. While the block-to-block levels of the acoustic source are observed to vary, the shape of the wave forms are consistent for a given experiment set, allowing for an average-level signal to be constructed.

For cases with no flow, a simple level check is applied to each block of data. Background fluctuations are observed to be on the order of tens of $\mathrm{mPa}$, while peak levels in the laser pulse source range between $20 \mathrm{~Pa}$ and 100 Pa with no flow and no seeding. However, for cases with significant flow noise a level check becomes inconsistent. While visual inspection of blocks shows obvious difference between data without a pulse and data with one, such an inspection process is intractable for the amount of data acquired. Instead, all of the blocks in the data set are ensemble averaged. Assuming a 10\% success rate for source occurrence (far below that actually realized with seeding), averaging 1000 blocks of data will yield a reduction in noise levels by 1000 , but in signal levels by only 100, yielding an effective boost in signal-to-noise ratio (SNR) of 10. This SNR-boosted waveform is then crosscorrelated with each block of data, and a peak-detection scheme applied to the cross-correlation at time bins near zero delay. Any block with a peak is retained as having a successful source occurrence, while any without is rejected.

In our previous research [14], a block rotation scheme was implemented due to small source movement within the facility. This step is unnecessary for these experiments. While some fluctuation in peak location within a given data set is observed for significant flow speeds, this fluctuation is found to be independent of whether or not flow seeding is present, and reduced in scale from the previous work. Photodetector output shows no such fluctuation, so source initiation time is consistent. It is not known whether the fluctuation is due to actual movement of the source due to facility vibration, or other flow-based phenomena such as low-frequency unsteadiness in the open jet shear layer.

A discrete Fourier transform is applied to the sampled data. The nature of the laser pulse source and ensembleaveraging process assures that the latter portion of each record is zero, so all records can be arbitrarily zero-padded to achieve a desired narrowband binwidth. In this case the data are padded such that the resultant binwidth is $16 \mathrm{~Hz}$, for comparison with acquired aeroacoustic data. For the single-microphone signal comparisons, a rectangular window is applied to gate the initial waveform and isolate it from any reflections. An example of this is shown in Figure 6. For all other analysis, no window function is applied. All microphone bins for each frequency are stacked

in a vector. The vector is multiplied by its conjugate transpose to build a CSM for that frequency, usable in 
beamforming through Equation (1). Diagonal removal is not applied, as self-noise is removed in the ensemble averaging process. The response functions required for steering vector correction are computed through direct division of the transformed data, as defined in Equation (14).

Aeroacoustic data are analyzed in a more traditional fashion. The 30 second records are broken into blocks $1 / 16^{\text {th }}$ of a second long for a resultant narrowband binwidth of $16 \mathrm{~Hz}$. Each block is multiplied by a Hanning window of appropriate length and transformed, and 75\% overlap is used in the analysis, yielding 996 effective block averages in the construction of the data CSM. Diagonal removal is applied to this data, slightly modifying the application of Equation (1) [3].

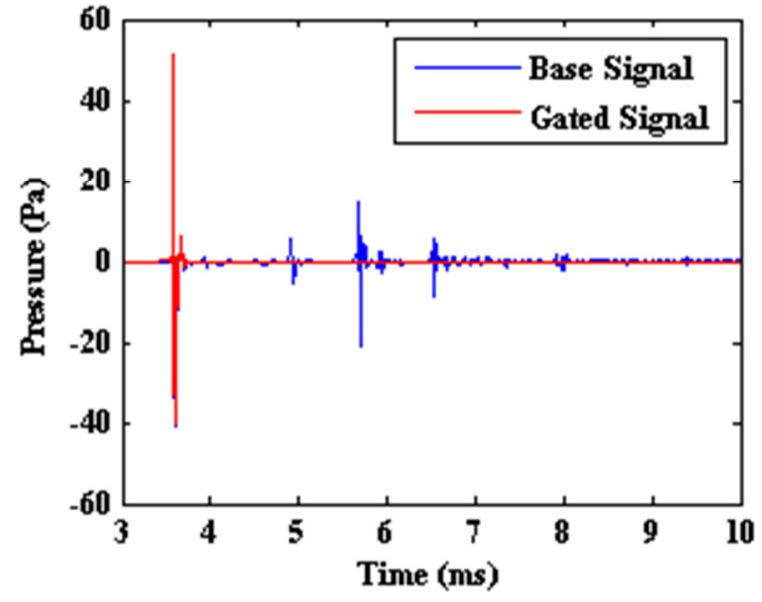

(a)

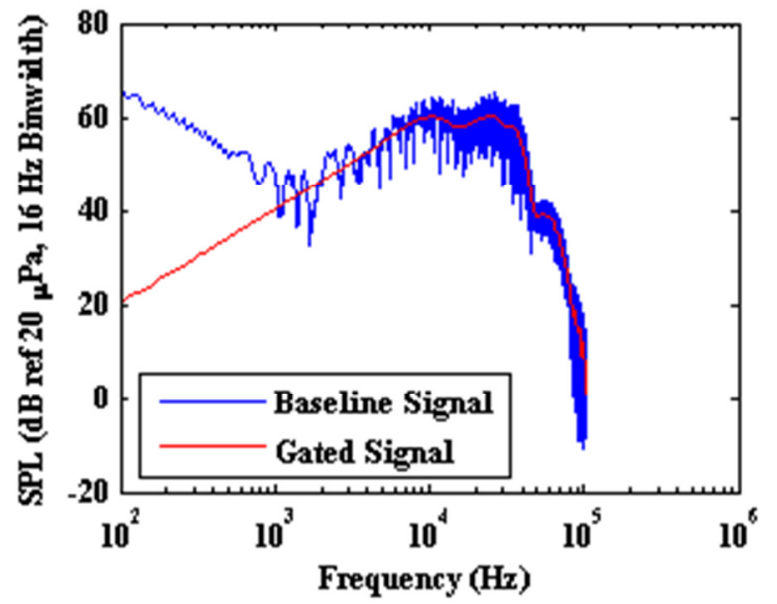

(b)

Figure 6. Example of signal gating in the (a) time and (b) frequency domains.

\section{E. Installation Configurations}

Several different experiment configurations are necessary to assess all of the different PSF properties of interest. These properties include variation with source location (testing for shift-invariance), inclusion of semi-reflective surfaces in a real experimental facility, effects of mean flow in an open jet facility, and inclusion of a real aeroacoustic model.

To assess the effects of source location, UFAFF's open-jet test section is lined with additional acoustic treatment to minimize any sources of reflection. For a source centered in the test section, this setup mimics that recommended by Dougherty [2]. The source is nominally located $1.13 \mathrm{~m}$ from the array face plane. Initial data are acquired for the source centered in the array-face coordinate system at $(0,0,1.13 \mathrm{~m})$. The array is then traversed within the facility such that the source's relative location to the array center is shifted. The array is moved to cover a 15-point grid in the $(x, y)$ plane, with $x$-coordinates of $0 \mathrm{~m}, \pm 0.279 \mathrm{~m}$, and $\pm 0.559 \mathrm{~m}$ and y-coordinates of $0 \mathrm{~m}$ and $\pm 0.279 \mathrm{~m}$. The z-plane remains unchanged. A photograph of this installation is shown with a coordinate system overlay and approximate laser source location is shown in Figure 7(a). Real facility effects are assessed by re-centering the array and removing the additional acoustic treatment visible on the sidewalls and in the test section inlet. The remaining sidewall material is acoustically absorbent, but has been observed to be semi-reflective at lower frequencies in previous research.

Mean flow effects are assessed with the array and model mounted in a vertical configuration. This is selected as the majority of current model configurations in UFAFF are designed for this orientation. Here, the array is located $1.13 \mathrm{~m}$ from the test section centerline, where the laser source is located. The laser optics direct through a small window installed flush in the lower test section sidewall. A photograph of this installation is shown in Figure 7(b). 
The facility is operated at quiescent conditions, as well as Mach numbers of $0.05,0.07,0.10,0.12,0.15$ and 0.17 . A $0.3048 \mathrm{~m}$ chord NACA 0012 airfoil is then mounted vertically in the facility, and the experiments repeated. The pulsed laser source is located approximately $0.038 \mathrm{~m}$ downstream of the airfoil trailing edge.

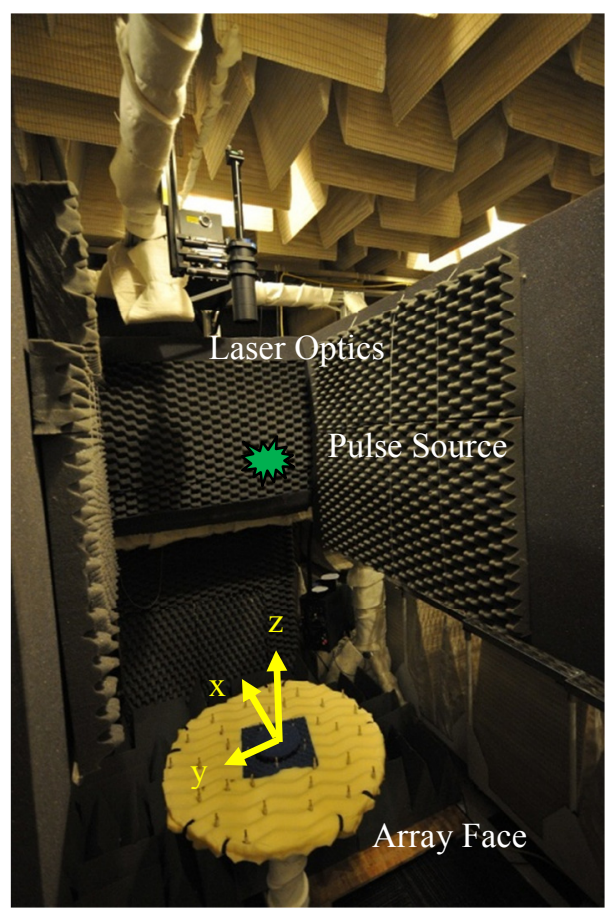

(a)

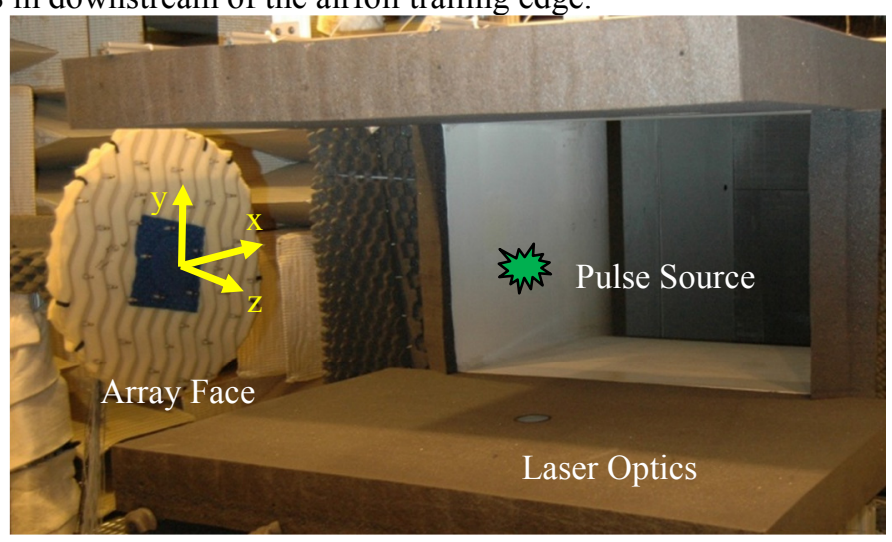

(b)

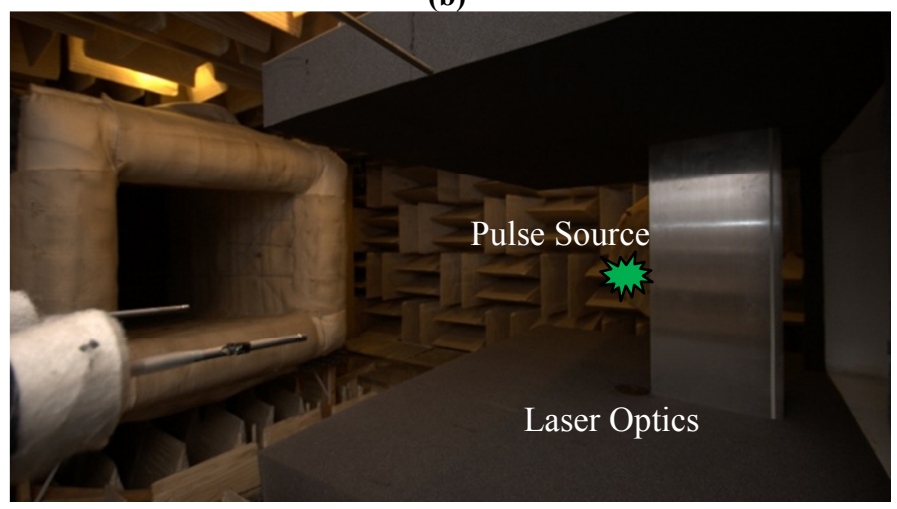

(c)

Figure 7. Photographs of experimental configurations (a) with anechoic treatment to evaluate source location effects, (b) for empty test section experiments with flow and (c) with a NACA 0012 airfoil installed.

\section{Results}

In this section, the initial source characteristics are first studied, followed by the effects of source location, sidewall changes, flow, and, finally, a real model installation.

\section{A. Acoustic Source Characteristics}

The laser pulse acoustic signal is first evaluated: (1) as a function of sampling rate to see how much of the true signal bandwidth may be lost to time-domain averaging and the PXI-4462 anti-aliasing filter; (2) as a function of installation to determine if scattering from the array is a significant component of the signal; and then (3) as a function of microphone grid and diameter in an attempt to estimate the potential bandwidth of the signal. These comparisons are currently qualitative, and do not account for individual microphone actuator response curves.

In Figure 8, the effect of system data acquisition on the signal of interest is evaluated. As stated above, the baseline signal is sampled at 204,800 samples per second, while the oversampled signal is sampled at 100 megasamples per second. As the source level is not consistent from pulse to pulse, the peak levels in the time series do not match up, resulting in an offset in the corresponding autospectra. Also, varying delay in the anti-aliasing filters of the different DAQ cards, along with variation in the speed of sound from one case to the next, leads to a difference in the observable relative delay between triggering and pulse occurrence from one case to the next. In the 
signal time series, the secondary and tertiary peaks in the pulse are missed for the baseline low sampling rate. In the spectrum, above $20 \mathrm{kHz}$ the baseline sampled signal appears to experience some reduction in level. While this does not reduce the utility of the signal above $20 \mathrm{kHz}$, as such attenuation should be universal across all channels for a given DAQ card and sampling rate, it does indicate that, for an ensemble-averaged signal, the baseline DAQ architecture does not adequately represent the full bandwidth available in the pulsed acoustic source.

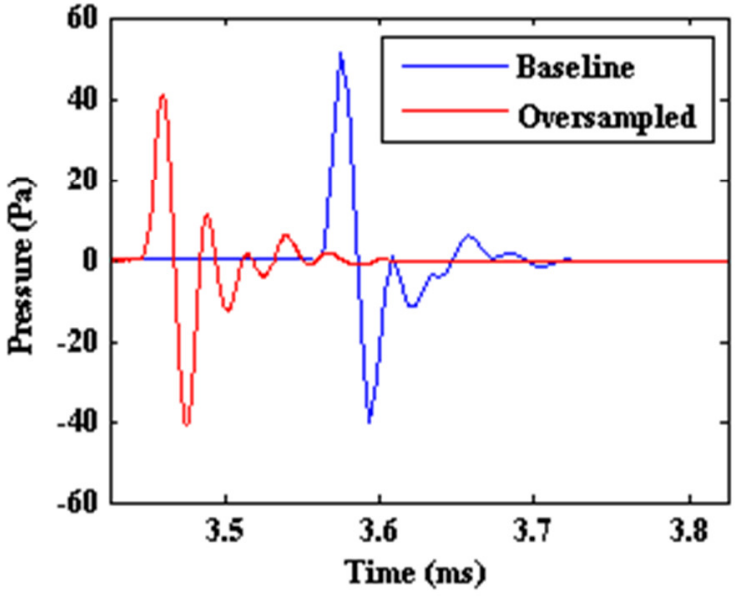

(a)

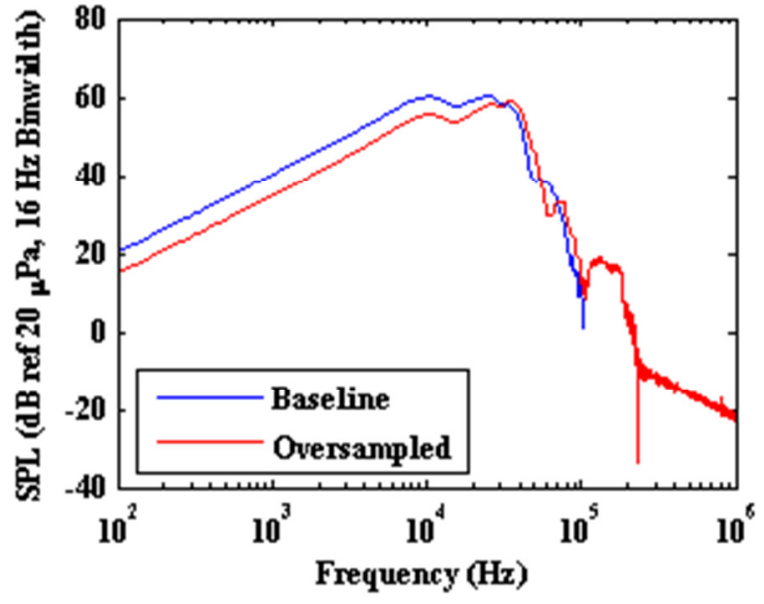

(b)

Figure 8. Evaluation of the effect of sampling rate and anti-aliasing filter on pulsed acoustic signal for the array-centered B\&K 4954 microphone; (a) time series comparison and (b) autospectrum comparison.

The various possible installation conditions for the microphones are now considered. For brevity in legends, each condition is assigned a case number. These cases are given in Table 1 . All of the cases aside from Case 1 are sampled at 100 megasamples per second. As the difference between Case 1 and Case 2 has already been addressed in Figure 8, it is omitted from the compilation of results in Figure 9. Subfigures (a) and (b), corresponding to a microphone installed in the center of the array illustrated in Figure 7(a), show the effect of the array installation on the observed signal. In the time domain, significant additional ripple is observable in the signal for the array frame case than for an equivalent free-field microphone. In the frequency domain, some variation in spectral shape is observable between $10 \mathrm{kHz}$ and $40 \mathrm{kHz}$. As with the previous comparison, speed of sound variations, and possibly differences in microphone impulse response functions from model-to-model, lead to a change in delay from trigger to observed peak arrival. Also, source levels are not consistent from case to case. However, the comparison clearly shows that above $10 \mathrm{kHz}$, the reference microphone at the array center is seeing a scattered field in addition to the incident acoustic pulse. While the described calibration method may account for scattering observed by array microphones, it does not account for scattering observed by the reference microphone, and so care must be taken with application.

Table 1. Listing of experimental cases for laser pulse source characterization.

\begin{tabular}{|c|c|c|c|c|}
\hline $\begin{array}{c}\text { Case } \\
\text { Number }\end{array}$ & $\begin{array}{c}\text { Microphone } \\
\text { Type }\end{array}$ & Installation & $\begin{array}{c}\text { Microphone } \\
\text { Grid }\end{array}$ & Sampling Rate \\
\hline 1 & B\&K 4954 (Free-Field) & Array Center & On & $204,800 \mathrm{samples} / \mathrm{sec}$ \\
\hline 2 & B\&K 4954 (Free-Field) & Array Center & On & $100 \mathrm{megasamples} / \mathrm{sec}$ \\
\hline 3 & B\&K 4939 (Free-Field) & Free Field & On & $100 \mathrm{megasamples} / \mathrm{sec}$ \\
\hline 4 & B\&K 4939 (Free-Field) & Free Field & Off & $100 \mathrm{megasamples} / \mathrm{sec}$ \\
\hline 5 & B\&K 4138 (Pressure-Field) & Free Field & On & $100 \mathrm{megasamples} / \mathrm{sec}$ \\
\hline
\end{tabular}

12

American Institute of Aeronautics and Astronautics 


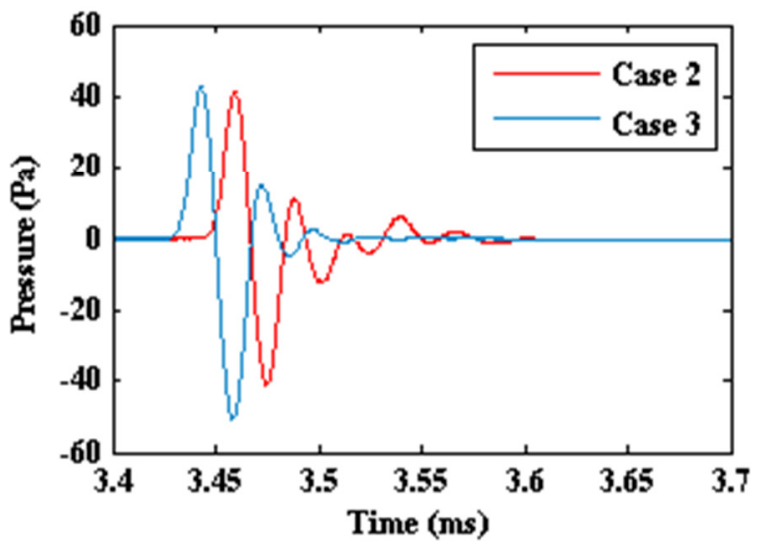

(a)

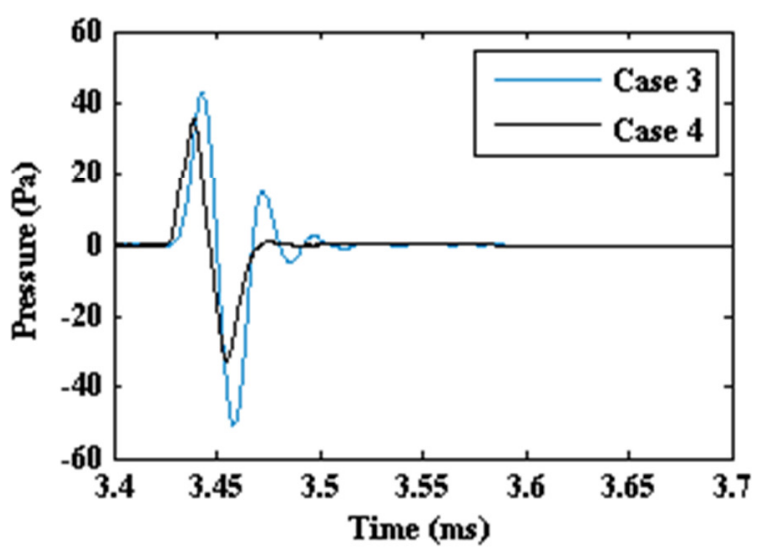

(c)

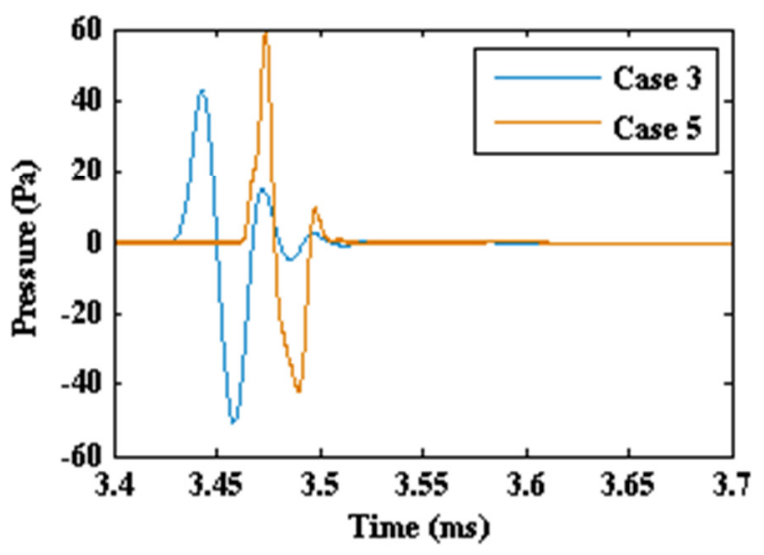

(e)

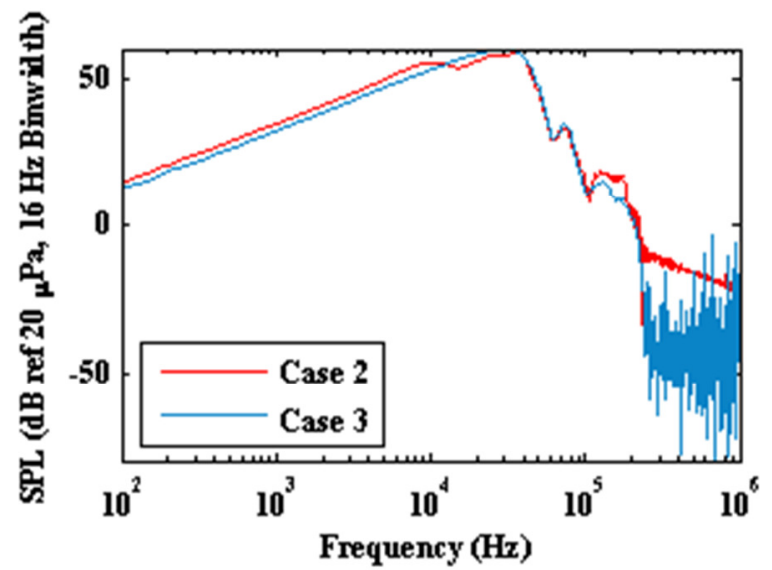

(b)

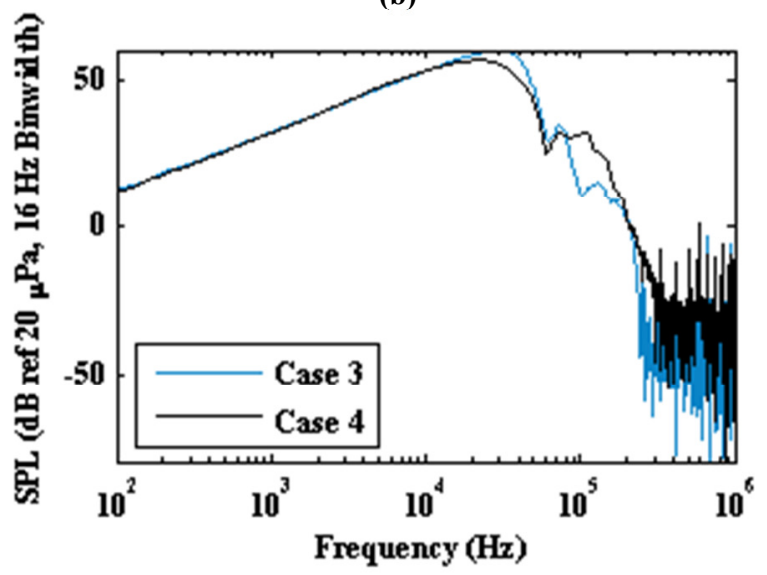

(d)

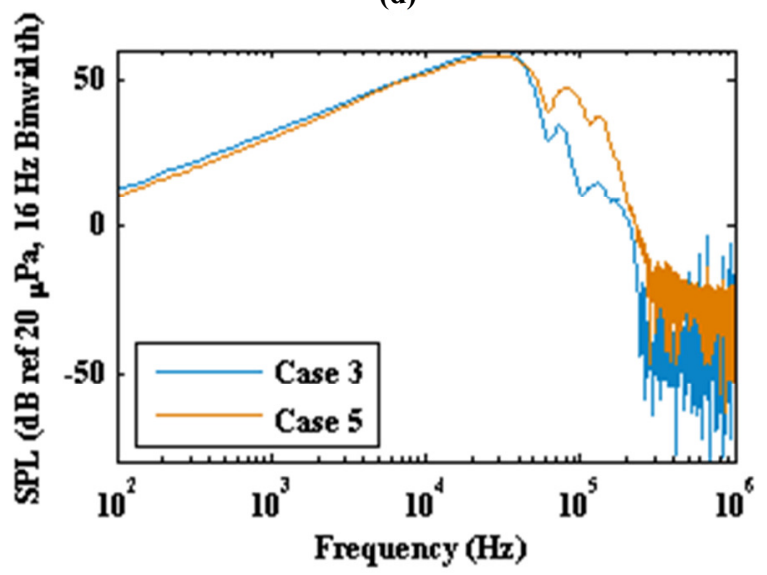

(f)

Figure 9. Evaluation of the effect of different microphone installations on the observed acoustic signal; (a) and (b) show the effect of the phased array frame in the time and frequency domain, (c) and (d) show the effect of the microphone grid, and (e) and (f) the effect of microphone diameter.

Figure 9(c) and (d) show the effect of the microphone grid on the observed signal. Here, the time series shows a significant reduction in ripple with the removal of the microphone grid. The spectra show that the grid adds gain to 
the acoustic signal in a band from below $20 \mathrm{kHz}$ up to $80 \mathrm{kHz}$, as expected from the grid correction data available from B\&K. However, the grid appears to significantly attenuate the signal upwards of $80 \mathrm{kHz}$.

Figure 9 (e) and (f) qualitatively show the effect of microphone size (and bandwidth) on the observed signal. This comparison is qualitative since free-field microphones include additional damping in the diaphragm response to compensate for free-field scattering, while pressure-field microphones do not [22]. This means that the expected observed output from a B\&K 4138 microphone should already show additional gain at high frequencies compared to a B\&K 4939 microphone, signal bandwidth aside. Nevertheless, the gain shown in the frequency domain of multiple orders of magnitude by using the smaller microphone is beyond any expected correction factor. Additionally, the sharpening of the initial peak in the time domain is indicative that the true signal bandwidth is well beyond that observable by a B\&K 4939 or B\&K 4138 microphone. The net result of this analysis shows that, while levels from experiment to experiment may not be completely repeatable, the pulsed laser source provides a high bandwidth signal with a consistent waveform shape (for a given physical installation), suitable for high frequency applications where precise phase information is desired.

\section{B. PSF as a Function of Source Location}

The array response in a clean acoustic installation, as shown in Figure 7(a), is now considered. The experimental PSFs are compared to theoretical PSFs for equivalent source locations. For this and all subsequent analyses, the discussion is limited to a case of $4 \mathrm{kHz}$. This frequency is selected as it is within a band unaffected by reference microphone scattering while having a small beamwidth given the installation dimensions of interest, while, for the NACA 0012 case, providing a data set where the airfoil trailing edge noise is dominant. All PSF plots are shown in a scale of $\mathrm{dB}$ normalized to peak levels.

The predicted and experimental PSF behaviors are shown in Figure 10. In all figures, the plotted coordinates correspond to those shown in the appropriate installation coordinate systems of Figure 7. Predicted PSFs are generated by simulating a monopole source at the desired location in space with a free-field propagation model, from Equation (12), for the array's design microphone coordinates. Both the simulated and experimental data are processed through Equation (1). For both cases, the PSFs are clearly not shift-invariant for these source-array combinations, as expected since source displacements within the scan plane are on the order of the array-to-scanplane distance. The overall behaviors of both are also similar, with regard to the displacement and distortion of the main lobe. However, specific details of the experimental data differ from the predictions. The shape of the experimental main lobes shows some additional asymmetry as compared to the theoretical responses. Sidelobe distributions also differ for each case.

\section{Minor Reflective Source}

The next case considered involves the removal of the additional acoustic treatment, as shown in Figure 7(a), lining the facility. The resultant test section configuration matches a more realistic installation, where only acoustic foam sidewalls are present similar to that shown in Figure 7(b), although still in a flyover configuration. Resultant beam maps for the theoretical free-space PSF and the measured PSF are shown in Figure 11. The sidewalls are illustrated in the figure, along with the contraction entrance into the facility. While the results do not show a perfect image source, the upper sidelobe is accentuated to a level beyond that visible for an equivalent experimental sourcearray configuration from Figure 10. This may be due to a mild reflection, indicating that, at $4 \mathrm{kHz}$, the facility does have some reflective behavior, which is also asymmetric. 


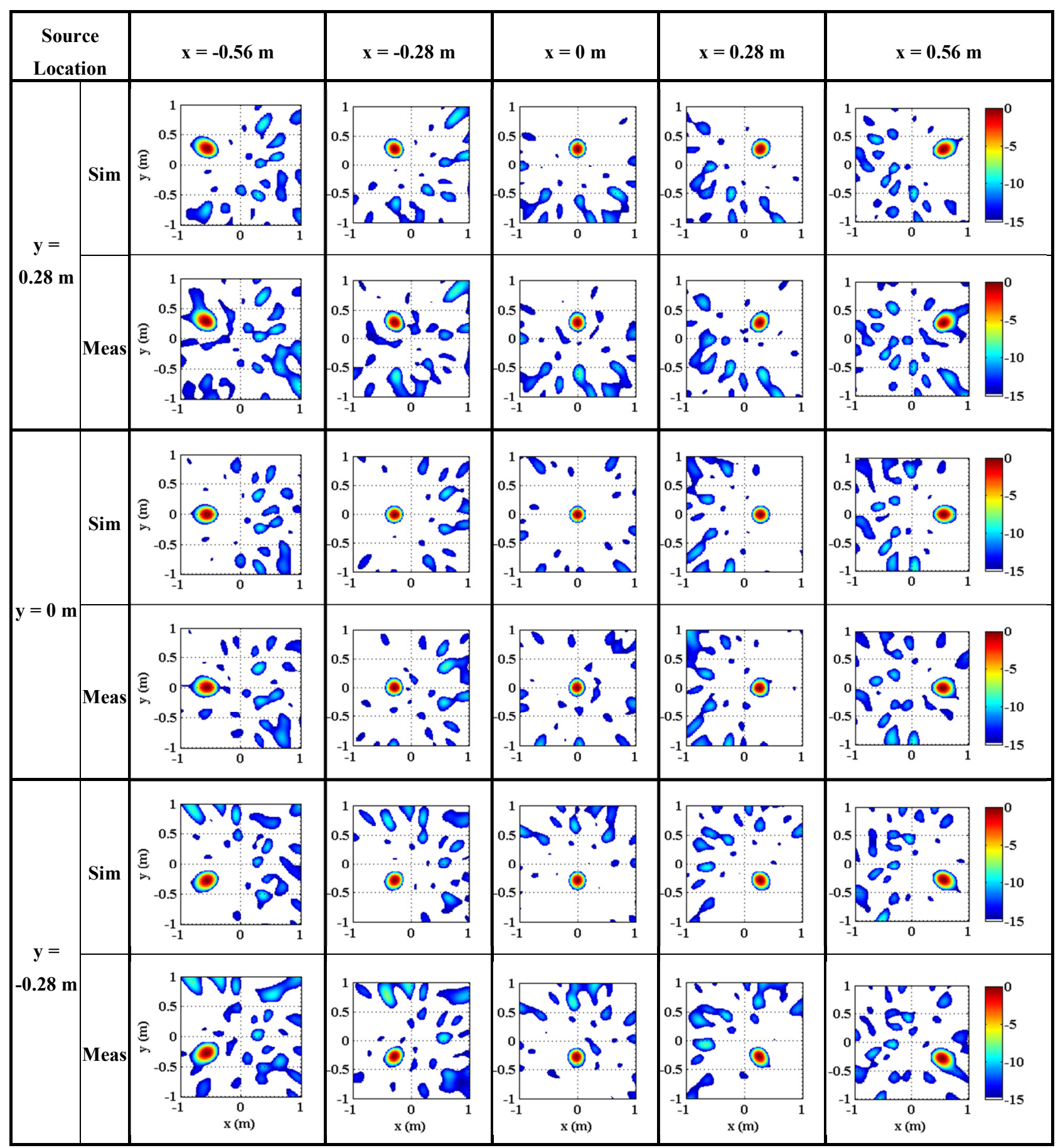

Figure 10. Simulated and measured PSF behavior at $4 \mathrm{kHz}$ for different source locations (normalized, $\mathrm{dB}$ ). 


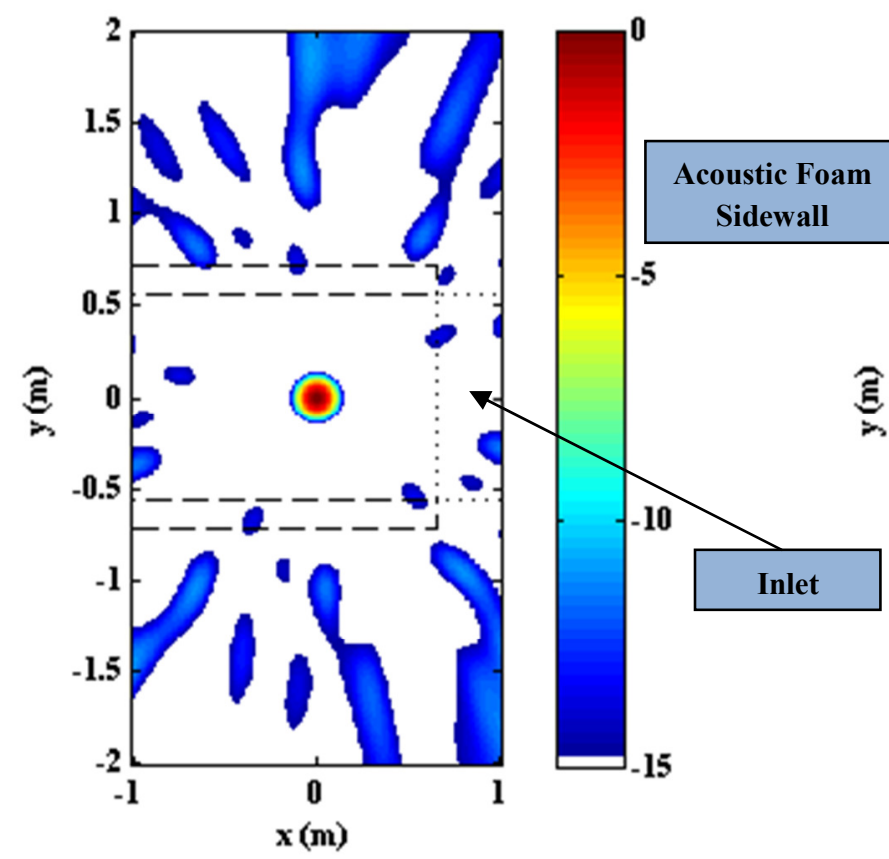

(a)

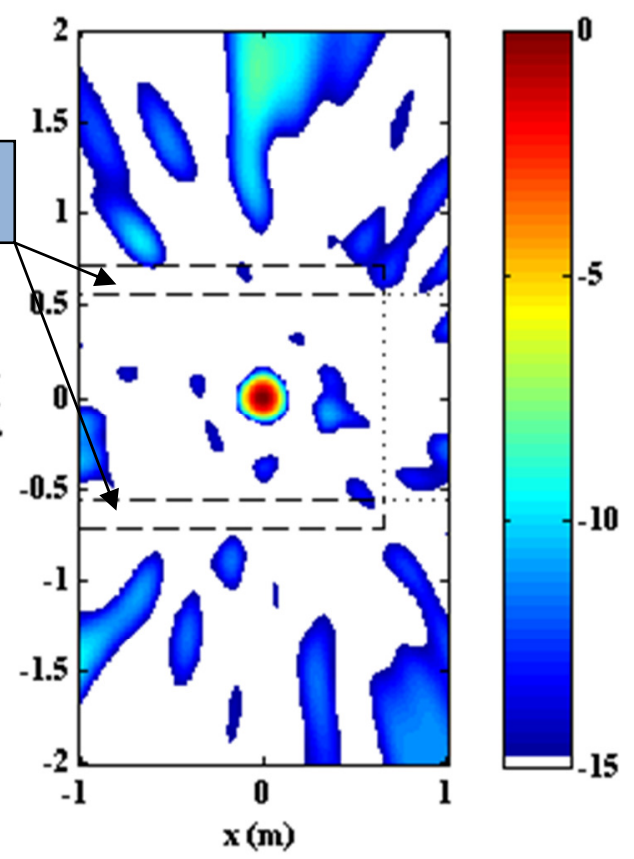

(b)

Figure 11. Comparison of (a) ideal free-space and (b) measured PSF at $4 \mathrm{kHz}$ for standard UFAFF configuration.

\section{Sideline Configurations - Effects of Flow and Model Installation}

The facility is reconfigured to a sideline installation as discussed previously. A legend of the symbols present on the beam maps is shown in Figure 12, to identify the overlays of sidewalls, the facility inlet, the test section jet collector and the NACA 0012 model. Previous research [14] has shown that Amiet's shear layer correction properly shifts data in UFAFF under regular, simple conditions such as these. As such, theoretical PSFs are not shown. Instead, PSF behavior in an empty test section is shown, followed by behavior with the NACA 0012 model.
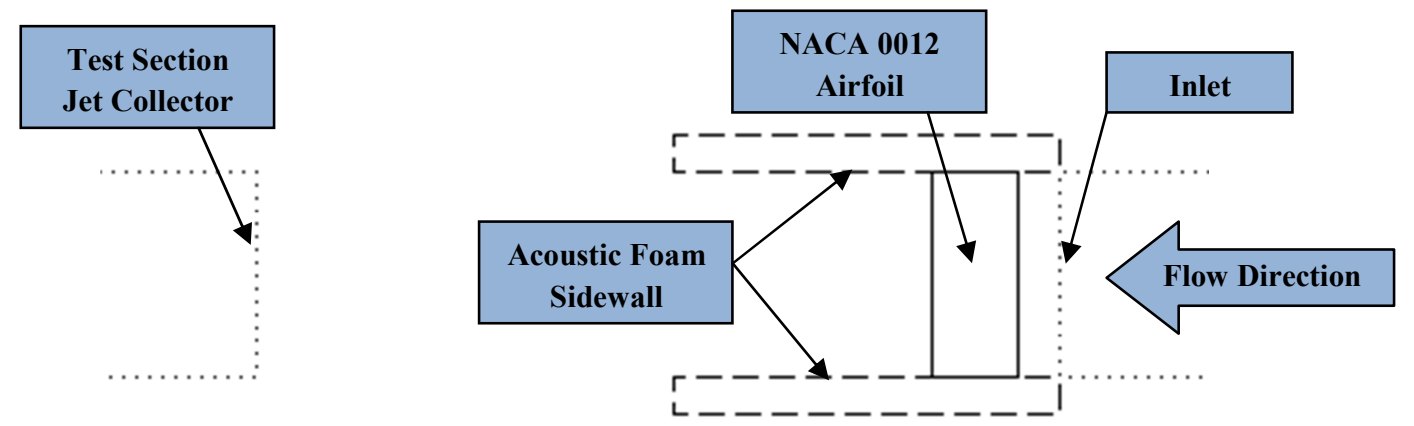

Figure 12. Legend of symbols used in sideline beam maps. 


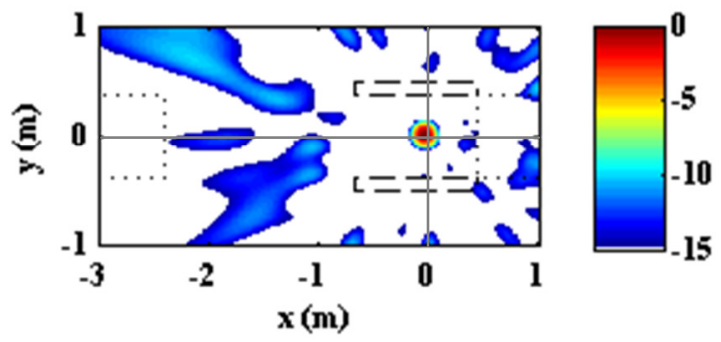

(a) No Flow

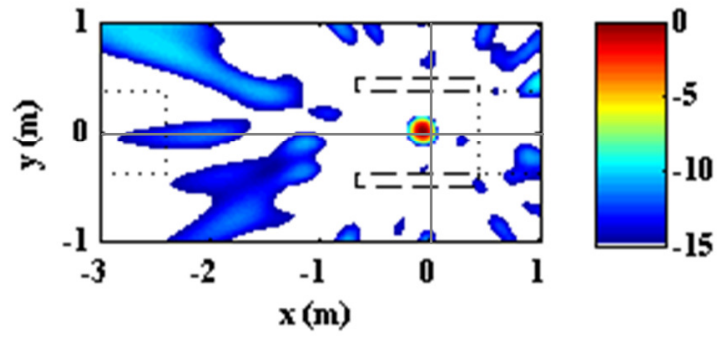

(b) Mach 0.05

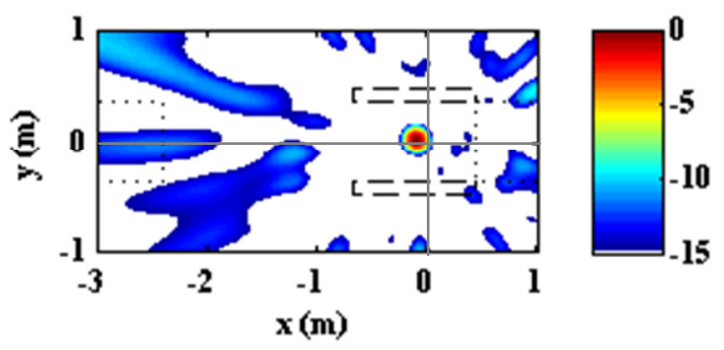

(d) Mach 0.10

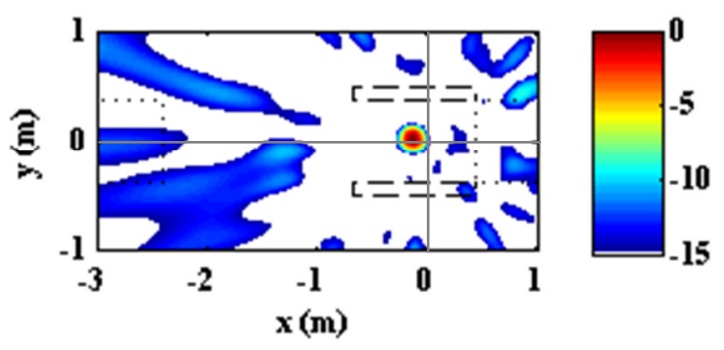

(f) Mach 0.15

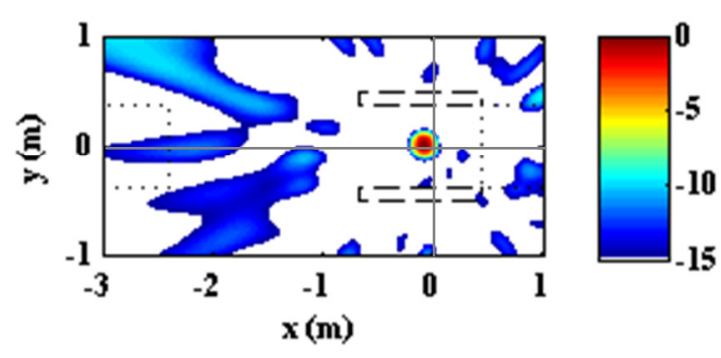

(c) Mach 0.07

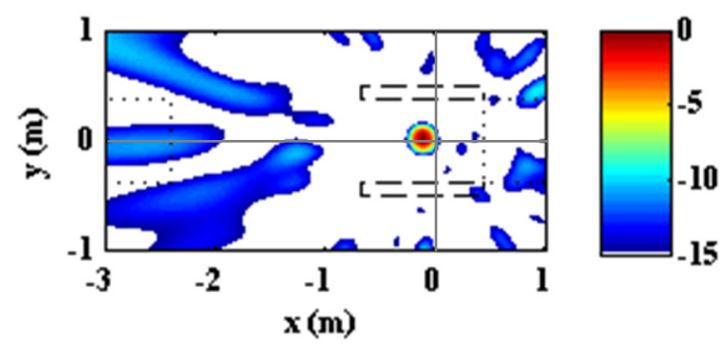

(e) Mach 0.12

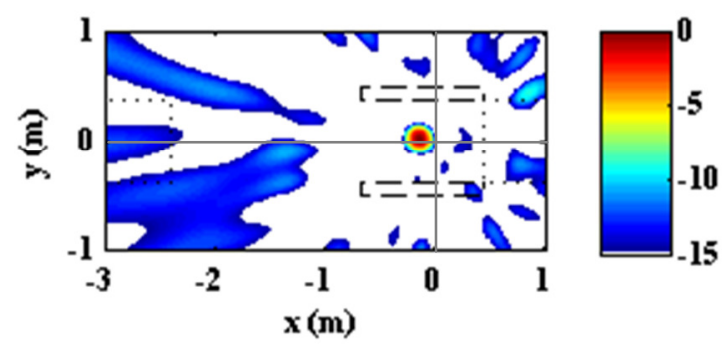

(g) Mach 0.17

Figure 13. PSF behavior at $4 \mathrm{kHz}$ for an empty test section configuration with varying flow Mach number, source location of $(-.03,0) \mathrm{m}$. Gray crosshairs added to aid visualization of apparent source position as a function of Mach number. 


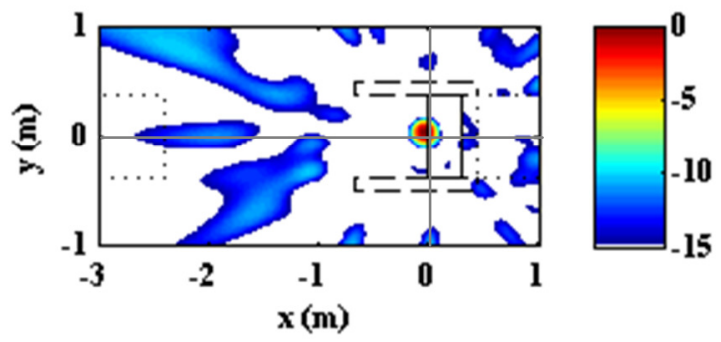

(a) No Flow

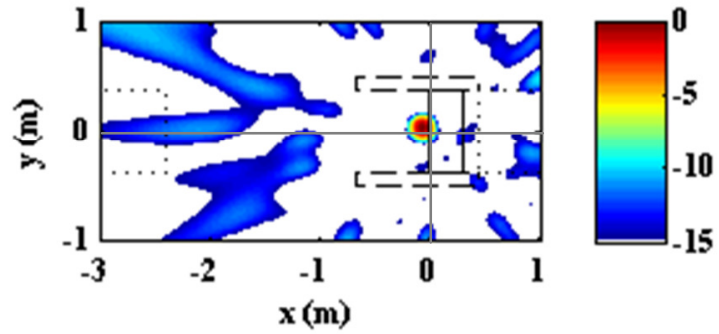

(b) Mach 0.05

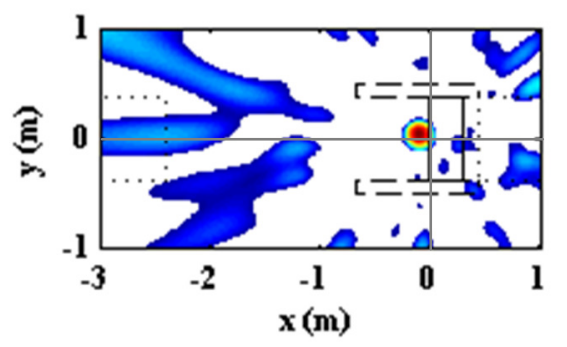

(d) Mach 0.10

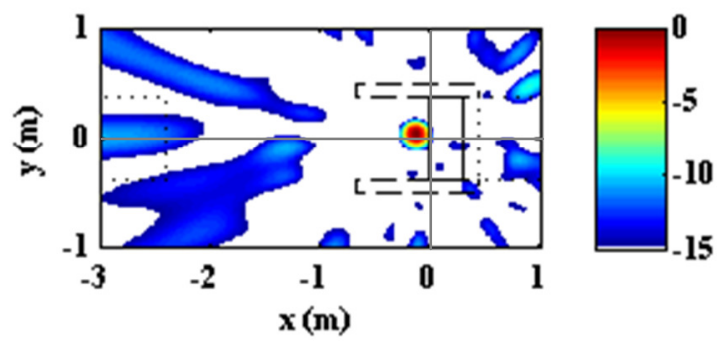

(f) Mach 0.15

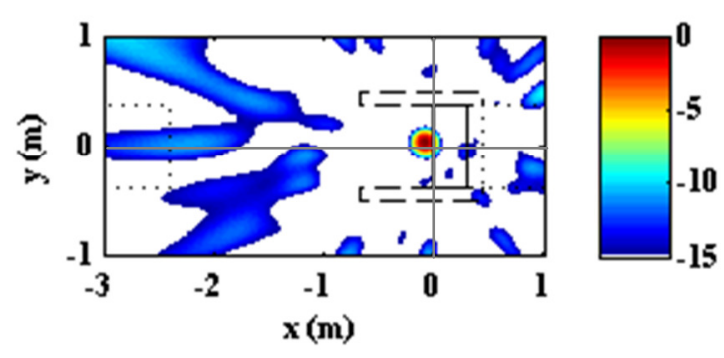

(c) Mach 0.07

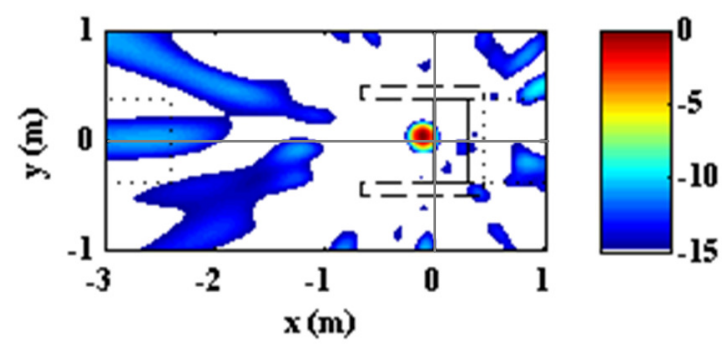

(e) Mach 0.12

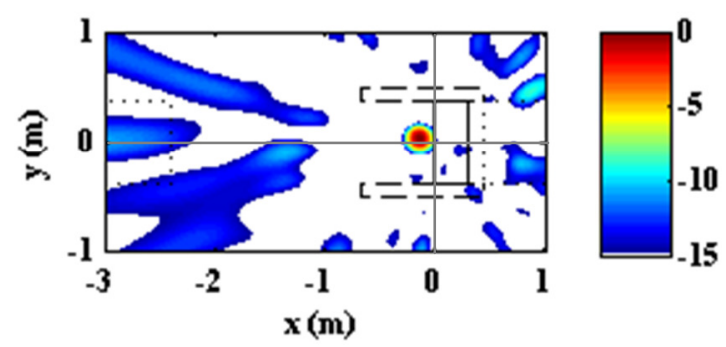

(g) Mach 0.17

Figure 14. PSF behavior at $4 \mathrm{kHz}$ for a NACA 0012 test section configuration $\left(0^{\circ} \mathrm{AoA}\right)$ with varying flow Mach number, source location of $(-.03,0) \mathrm{m}$. Gray crosshairs added to aid visualization of apparent source position as a function of Mach number. 
Figure 13 shows the PSF as a function of Mach number for the empty test section configuration shown in Figure 7(b). Figure 14 shows the PSF as a function of Mach number for the airfoil configuration shown in Figure 7(c). Both of these cases show extremely similar behavior. The array PSF, while slightly skewed, appears to have a Mach number invariant shape. Also, no reflections are visible from the sidewalls, inlet or jet collector assemblies. Of note, the apparent source location is $\sim 0.04 \mathrm{~m}$ higher along the $\mathrm{y}$-axis than the true source location. This is indicative of array misalignment in the experiment.

Misalignment aside, these results raise the question as to why such measurements may be necessary for these experiments, as opposed to using a free-space steering vector and simply applying Amiet's correction. The answer to this comes from the response functions computed in Section II. For a true, free-space source, the phase of the response function between the reference microphone and any array microphone should be linear as a function of frequency, representing an ideal delay. As Amiet's method simply modifies the delay, the modified response function should still be linear as a function of frequency. To evaluate the validity of this, sample response functions between the reference microphone and the $40^{\text {th }}$ microphone in the array (located on the outer array ring) are shown in Figure 15.

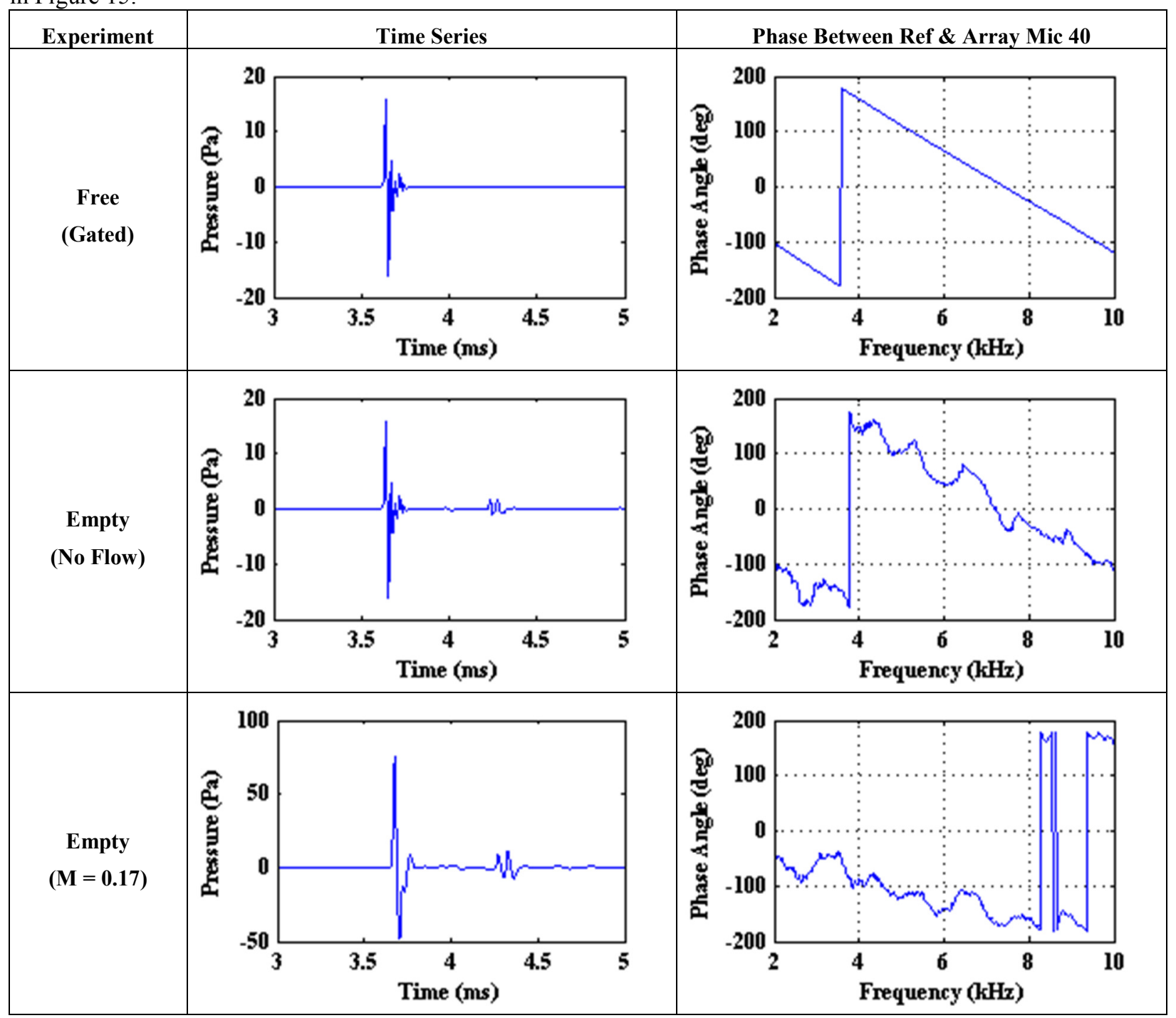

Figure 15. Evaluation of response functions for different installation conditions. 
From these plots, it is clear that while the clean signal does show a linear phase response, the experimental conditions for the real facility without and with flow do not. The secondary structure in the second and third time series corresponds to the appropriate delay time for a reflection from the acoustic foam sidewalls. While the reflections seen in the time series do not manifest as noticeable sources in previously-evaluated beam maps, they do appear to have a significant effect on the phase behavior between microphones. The deviation in these phase plots could lead to serious errors in levels computed from beamformer outputs, and would not be accounted for using a simple shear layer correction method with a free-space steering vector.

\section{E. Corrections Applied to Aeroacoustic Data}

Finally, the laser pulse data is used in an attempt to correct an aeroacoustic data set. Specifically, data are acquired from the NACA 0012 airfoil at a Mach number of 0.17 and an angle of attack of $0^{\circ}$. The data are analyzed in Figure 16 first with no corrections, then with Amiet's planar shear layer correction method, and finally with the laser pulse data, applied as discussed in Section II. The experimental setup is identical to that shown in Figure 12, but outer regions of the test section are omitted to provide more detail of the airfoil region. Diagonal removal is applied [2-3]. Decibel levels in the colormap are computed with respect to a reference level of 2e-5 Pa.

(a)
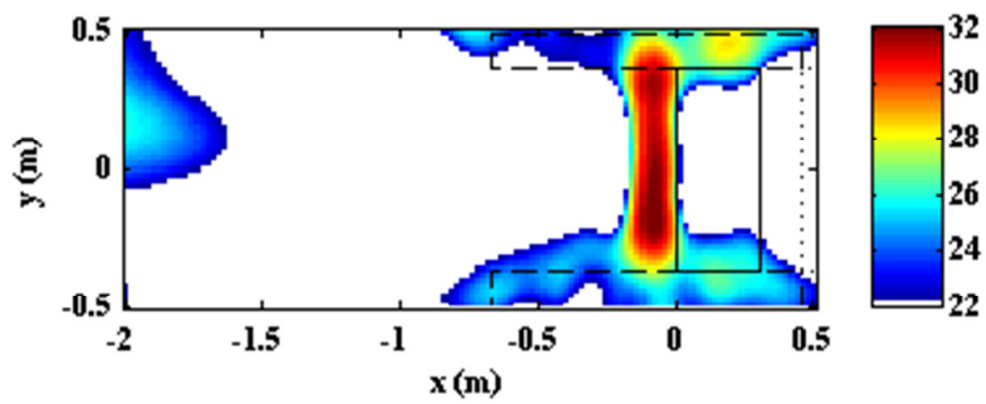

(b)

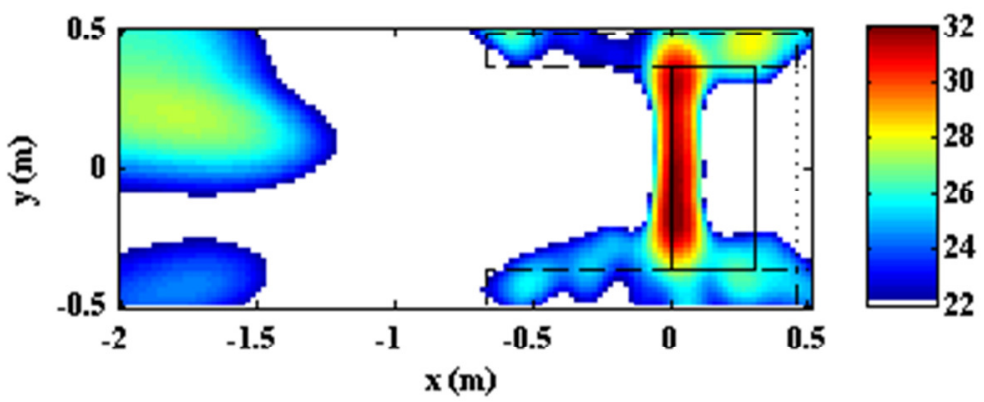

(c)

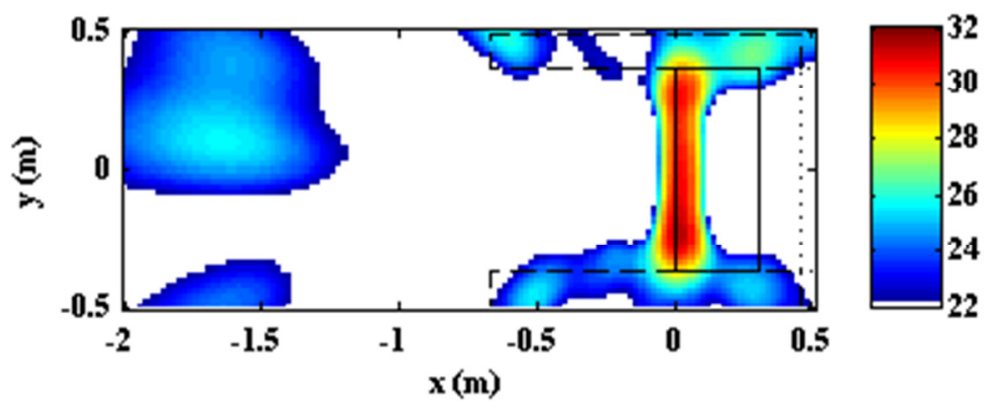

Figure 16. Experimental aeroacoustic data for a NACA 0012 airfoil at Mach $0.17,4 \mathrm{kHz}$, (a) uncorrected, (b) corrected with Amiet's method for a planar shear layer and (c) corrected with pulsed calibration data. 
The baseline, uncorrected case in subfigure (a) shows the expected behavior for this flow condition, given a misaligned array which leads to a vertical offset in the beam map. The trailing edge noise source is dominant, but is shifted downstream in the beam map. Amiet's correction method, (b), restores it to the vicinity of the trailing edge. However, Amiet's method does nothing with regard to the noise sources along the sidewalls, whether they be scrubbing noise, sidelobes, or out-of-plane noise sources. It also does not account for the array misalignment. Furthermore, it enhances the downstream sidelobe. The experimentally-corrected steering vectors, like Amiet's correction method, properly restore the trailing edge noise source to the model trailing edge, and corrects for the apparent alignment issue. However, the method shown in (c) does so with less enhancement of the downstream sidelobe, and actually shows mitigation of the extraneous sources along the test section sidewall. Additionally, the experimental correction method appears to sharpen the trailing edge noise source in the beam map as compared to the Amiet-corrected and uncorrected data. Overall level ranges within the subfigures are all of similar scale.

\section{Conclusions and Future Work}

A non-intrusive point source is used to measure the PSF of a broadband, aeroacoustic phased microphone array under varying experimental conditions. To generate the source, a Nd:YAG laser is focused to a point in space and pulsed at $1 \mathrm{~Hz}$. The repetitive acoustic source is high bandwidth and consistent in waveform shape, although overall pulsed levels vary from block to block. Signals are ensemble-averaged to focus on the deterministic, periodic laser pulsing and reject background and model flow noise. Facility conditions are varied from an anechoic-like treatment to a true experimental configuration with an airfoil model and low Mach number flow.

The results shown indicate that the pulsed laser source is a valid way to measure an array's PSF. The source has sufficient bandwidth to cover the entire acoustic measurement range of interest and allows for characterization of installation realities, such as microphone-array-frame scattering, which may not otherwise be accounted for in an experiment. Under clean conditions, the beamwidth of the experimental data matches the design beamwidth of the array for a given frequency. Sidelobe distributions differ, but not by a significant amount in most cases considered to date, and may be due to errors in microphone locations. The measurement method is capable of detecting partial reflections in a given configuration. Also, it provides an alternative to applying a shear layer correction technique, at least for near shift-invariant measurement conditions, when a mean shear layer is present in an open jet measurement configuration.

Subsequent work will involve additional investigation of coherent reflection characteristics with various boundary conditions more closely approaching a hard-walled boundary condition. Also, additional aeroacoustic measurements should be considered where the region of interest is no longer shift-invariant, such that a shift-based correction can be compared with an Amiet-based correction. The effect of the correction method on integrated level uncertainties must be considered, as well as direct incorporation into deconvolution beamforming.

\section{Acknowledgements}

The authors acknowledge the financial support provided by the Florida Center for Advanced Aero-Propulsion. 


\section{References}

[1] D.H. Johnson and D.E. Dudgeon, Array Signal Processing: Concepts and Techniques, Prentice Hall, 1993.

[2] R.P. Dougherty, "Beamforming in Acoustic Testing," in Aeroacoustic Measurements, T.J. Mueller, Ed., Springer-Verlag, Berlin, Heidelberg \& New York, 2002.

[3] T.F. Brooks and W.M. Humphreys, "A Deconvolution Approach for the Mapping of Acoustic Sources (DAMAS) Determined from Phased Microphone Arrays," Journal of Sound and Vibration, Vol. 294, pp. 856-879, 2006.

[4] R.P. Dougherty, "Extensions of DAMAS and Benefits and Limitations of Deconvolution in Beamforming," AIAA Paper 2005-2961, $11^{\text {th }}$ AIAA/CEAS Aeroacoustics Conference, Monterey, CA, May 2005.

[5] K. Ehrenfried and L. Koop, "Comparison of Iterative Deconvolution Algorithms for the Mapping of Acoustic Sources,” AIAA Journal, Vol. 45, No. 7, pp. 1584-1595, 2007.

[6] P. Sijtsma, "CLEAN Based on Spatial Source Coherence," International Journal of Aeroacoustics, Vol. 6, No. 4, pp. 357-374, 2007.

[7] T. Yardibi, J. Li, P. Stoica, L. Cattafesta III, "Sparsity Constrained Deconvolution Approaches for Acoustic Source Mapping," Journal of the Acoustical Society of America, Vol. 123, No. 5, pp. 2631-2642, 2008.

[8] P.A. Ravetta, R.A. Burdisso and W.F. Ng, "Noise Source Localization and Optimization of Phased-Array Results", AIAA Journal, Vol. 47, No. 11, pp. 2520-2533, 2009.

[9] T. Yardibi, J. Li, P. Stoica, N. Zawodny and L. Cattafesta III, "A Covariance-Fitting Approach for Correlated Acoustic Source Mapping," Journal of the Acoustical Society of America, Vol. 127, No. 5, pp. 29202931, 2010.

[10] T. Suzuki, "DAMAS2 Using a Point-Spread Function Weakly Varying in Space," AIAA Journal, Vol. 48, No. 9, pp. 2165-2169, 2010.

[11] W.M. Humphreys, T.F. Brooks, W.W. Hunter Jr. and K.R. Meadows, "Design and Use of Microphone Directional Arrays for Aeroacoustic Measurements," AIAA Paper 98-0471, 36th AIAA Aerospace Sciences Meeting \& Exhibit, Reno, NV, January 1998.

[12] S. Oerlemans and P. Sijtsma, "Effects of Wind Tunnel Side-Plates on Airframe Noise Measurements with Phased Arrays,” NLR-TP-2000-169, 2000.

[13] B.A. Fenech and K. Takeda, "Toward More Accurate Beamforming Levels in Closed-Section Wind Tunnels via De-Reverberation," AIAA Paper 2007-3431, $13^{\text {th }}$ AIAA/CEAS Aeroacoustics Conference, Rome, May 2007.

[14] C. Bahr, N. Zawodny, T. Yardibi, F. Liu, D. Wetzel, B. Bertolucci and L. Cattafesta III, "Shear Layer Time-Delay Correction using a Non-Intrusive Acoustic Point Source," International Journal of Aeroacoustics, to appear, 2010.

[15] T. Yardibi, C. Bahr, N. Zawodny, F. Liu, L. Cattafesta III and J. Li, "Uncertainty Analysis of the Standard Delay-and-Sum Beamformer and Array Calibration," Journal of Sound and Vibration, Vol. 329, pp. 2654-2682, 2010.

[16] T.F. Brooks, W.M. Humphreys and G.E. Plassman, "DAMAS Processing for a Phased Array Study in the NASA Langley Jet Noise Laboratory," AIAA Paper 2010-3780, $16^{\text {th }}$ AIAA/CEAS Aeroacoustics Conference, Stockholm, Sweden, June 2010.

[17] D.T. Blackstock, "Spherical Waves," Fundamentals of Physical Acoustics, John Wiley and Sons, Inc., New York, 2000.

[18] Q. Qin and K. Attenborough, "Characteristics and Application of Laser-Generated Acoustic Shock Waves in Air," Applied Acoustics, Vol. 65, pp. 325-240, 2004. 
[19] CVI Melles Griot, "Gaussian Beam Optics,"

https://cvimellesgriot.com/Products/Documents/TechnicalGuide/Gaussian-Beam-Optics.pdf, (last accessed April 2011), 2009.

[20] D. Wetzel, “An Experimental Investigation of Circulation Control Acoustics," Ph.D. Dissertation, Mechanical and Aerospace Engineering, University of Florida, 2011.

[21] J.R. Underbrink, "Practical Considerations in Focused Array Design for Passive Broad-Band Source Mapping Applications," M.S. Thesis, Acoustics Dept., The Pennsylvania State University, State College, PA, 1995.

[22] Bruel \& Kjær, "Chapter 2: Microphone Theory," Microphone Handbook Vol. 1: Theory, BE 1447-11, Nærum, Denmark, July 1996. 